\title{
APPLICATION OF SRIVASTAVA-ATTIYA OPERATOR TO THE GENERALIZATION OF MOCANU FUNCTIONS
}

\section{KHALIDA INAYAT NOOR AND SHUJAAT ALI SHAH*}

\author{
COMSATS University Islamabad, Pakistan
}

*Corresponding author: shahglike@yahoo.com

\begin{abstract}
In this paper we introduce certain subclasses of analytic functions by applying Srivastava-Attiya operator. Our main purpose is to derive inclusion results by using concept of conic domain and subordination techniques. We also deduce some new as well as well-known results from our investigations.
\end{abstract}

\section{INTRODUCTION}

Let $\chi$ denotes the class of analytic functions $f(z)$ in the open unit disk $\mho=\{z:|z|<1\}$ such that

$$
f(z)=z+\sum_{n=2}^{\infty} a_{n} z^{n} .
$$

Subordination of two functions $f$ and $g$ is denoted by $f \prec g$ and defined as $f(z)=g(w(z))$, where $w(z)$ is schwarz function in $\mho$. Let $S, S^{*}$ and $C$ denotes the subclasses of $\chi$ of univalent functions, starlike functions and convex functions respectively. For $0 \leq \delta<1, S^{*}(\delta)$ and $C(\delta)$ are the subclasses of $S$ of functions $f$ satisfies;

$$
\frac{z f^{\prime}(z)}{f(z)} \prec \frac{1+(1-2 \delta) z}{1-z}, \quad(z \in \mho)
$$

Received 2019-04-24; accepted 2019-05-27; published 2019-07-01.

2010 Mathematics Subject Classification. 30C45, 30C55.

Key words and phrases. Srivastava-Attiya operator; Mocanu functions; conic domains.

(C)2019 Authors retain the copyrights of their papers, and all open access articles are distributed under the terms of the Creative Commons Attribution License. 


$$
\frac{\left(z f^{\prime}(z)\right)^{\prime}}{f^{\prime}(z)} \prec \frac{1+(1-2 \delta) z}{1-z}, \quad(z \in \mho \delta),
$$

respectively. Mocanu [13] introduced the class $M_{\alpha}$ of $\alpha$-convex functions $f \in S$ satisfies;

$$
\left((1-\alpha) \frac{z f^{\prime}(z)}{f(z)}+\alpha \frac{\left(z f^{\prime}(z)\right)^{\prime}}{f^{\prime}(z)}\right) \prec \frac{1+z}{1-z},
$$

where $\alpha \in[0,1], \frac{f(z)}{z} f^{\prime}(z) \neq 0$. and $z \in \mho$. We see that $M_{0}=S^{*}$ and $M_{1}=C$. This class is vastly studied by several authors. See $[4,15,17-19]$. For $k \in[0, \infty)$, Kanas and Wisniowska [8,9] introduced the classes $k-U C V$ of k-uniformly convex functions and $k-S T$ of $\mathrm{k}$-starlike functions. The analytic conditions for these classes are given [6-9] as;

$$
\begin{gathered}
k-U C V=\left\{f \in \mathbf{S}: \operatorname{Re}\left(1+\frac{z f^{\prime \prime}(z)}{f^{\prime}(z)}\right)>k\left|\frac{z f^{\prime \prime}(z)}{f^{\prime}(z)}\right|\right\}, \quad(z \in \mho) . \\
k-S T=\left\{f \in \mathbf{S}: \operatorname{Re}\left(\frac{z f^{\prime}(z)}{f(z)}\right)>k\left|\frac{z f^{\prime}(z)}{f(z)}-1\right|\right\}, \quad(z \in \mho) .
\end{gathered}
$$

We can rewrite the above relations easily as;

$$
\operatorname{Re}(p(z))>k|p(z)-1|
$$

where $p(z)=1+\frac{z f^{\prime \prime}(z)}{f^{\prime}(z)}$ or $p(z)=\frac{z f^{\prime}(z)}{f(z)}$. It is clear that $p(\mho)$ is conic domain defined as;

$$
\Omega_{k}=\{w \in \mathbb{C}: \operatorname{Re}(w)>k|w-1|\}
$$

or

$$
\Omega_{k}=\left\{u+i v: u>k \sqrt{(u-1)^{2}+v^{2}}\right\}, \quad(0 \leq k<\infty) .
$$

These conic domains are being studied by several authors. See [2,6,14,16]. Sokol and Nonukawa [23] introduced the class defined as;

$$
M N=\left\{f \in S: \operatorname{Re}\left(1+\frac{z f^{\prime \prime}(z)}{f^{\prime}(z)}\right)>\left|\frac{z f^{\prime}(z)}{f(z)}-1\right|\right\}, \quad(z \in \mho \delta) .
$$

It is obvius that $M N \subset C$. Recently S. Sivasubramanian et al. [22] extend the Sokol and Nonukawa's work in terms of conic domains. They introduced a new class $k-M N$ of functions $f \in S$ such that

$$
\left.\operatorname{Re}\left(1+\frac{z f^{\prime \prime}(z)}{f^{\prime}(z)}\right)>k\left|\frac{z f^{\prime}(z)}{f(z)}-1\right|, \quad(z \in \mho)\right) .
$$

In motivation of the work [23], A. Rasheed et al. [21], introduced an interesting class $k-U M_{\alpha}(0 \leq \alpha \leq 1)$ of functions $f \in S$ such that 


$$
\operatorname{Re}\left[(1-\alpha) \frac{z f^{\prime}(z)}{f(z)}+\alpha \frac{\left(z f^{\prime}(z)\right)^{\prime}}{f^{\prime}(z)}\right]>k\left|\frac{z f^{\prime}(z)}{f(z)}-1\right|, \quad(z \in \mho) .
$$

Obviously, we can see $k-U M_{1}=k-M N$ and $1-U M_{1}=M N$.

We recall a Hurwitz-Lerch Zeta function $\Phi(s, b ; z)[25]$ defined by

$$
\Phi(s, a ; z)=\sum_{n=2}^{\infty} \frac{z^{n}}{(n+b)^{s}}
$$

$\left(b \in \mathbb{C} \backslash \mathbb{Z}_{0}^{-} ; s \in \mathbb{C}\right.$ when $|z|<1 ; \operatorname{Re}(s)>1$ when $\left.|z|=1\right)$,

where $\mathbb{C}$ and $\mathbb{Z}_{0}^{-}$denotes the set of complex numbers and the set of negative integers respectively.

Srivastava and Attiya [24] introduced the linear operator $J_{s, b}: \chi \rightarrow \chi$ defined in terms of the convolution ( or Hadamard product), by

$$
J_{s, b} f(z)=G_{s, b}(z) * f(z)
$$

where

$$
G_{s, b}(z)=(1+b)^{s}\left[\Phi(s, b ; z)-b^{s}\right],
$$

with $z \in \mho, b \in \mathbb{C} \backslash \mathbb{Z}_{0}^{-}$and $s \in \mathbb{C}$. Therefore, using (1.13) to (1.15), we have

$$
J_{s, b} f(z)=z+\sum_{n=2}^{\infty}\left(\frac{1+b}{n+b}\right)^{s} a_{n} z^{n},
$$

where $z \in \mho, b \in \mathbb{C} \backslash \mathbb{Z}_{0}^{-}$and $s \in \mathbb{C}$.

The srivastava-Attiya operator generalizes the integral operators introduced by Alexandar [1], Libera [10], Bernardi [3] and Jung et al. [5].

In 2007, Raducanu and Srivastava [20] introduced and studied the class $S_{s, b}^{*}(\delta)$ of functions $f \epsilon \chi$ satisfies $J_{s, b} f(z) \epsilon S^{*}(\delta)$.

Now by using concepts of conic domains and Srivastava-Attiya integral operator, we introduce new classes as following.

Definition 1.1. Let $k \in[0, \infty)$ and $\alpha, \beta \in[0,1]$. Then $f \in k-U M(\alpha, \beta)$ if and only if

$$
\operatorname{Re}\left[(1-\alpha) \frac{z f^{\prime}(z)}{f(z)}+\alpha \frac{\left(z f^{\prime}(z)\right)^{\prime}}{f^{\prime}(z)}\right]>k\left|(1-\beta) \frac{z f^{\prime}(z)}{f(z)}+\beta \frac{\left(z f^{\prime}(z)\right)^{\prime}}{f^{\prime}(z)}-1\right|, \quad(z \in \mho) .
$$

Some of the special cases are given below and we refer to $[8,9,21-23]$.

\section{Special cases:}

(i) For $\beta=0$, the class $k-U M(\alpha, \beta)$ reduces to the class $k-U M_{\alpha}$. See [21].

(ii) For $\alpha=1$ and $\beta=0$, the class $k-U M(\alpha, \beta)$ reduces to the class $k-M N$. See [22].

(iii) For $\alpha=1, \beta=0$ and $k=1$, the class $k-U M(\alpha, \beta)$ reduces to the class $M N$. See [23].

(iv) For $\alpha=1$ and $\beta=1$, the class $k-U M(\alpha, \beta)$ reduces to the class $k-U C V$. See [9]. 
(v) For $\alpha=0$ and $\beta=0$, the class $k-U M(\alpha, \beta)$ reduces to the class $k-S T$. See [8].

Definition 1.2. Let $\alpha, \beta \in[0,1], k \in[0, \infty), b \in \mathbb{C} \backslash \mathbb{Z}_{0}^{-}$and $s \in \mathbb{C}$. Then $f \epsilon k-U M_{b}^{s}(\alpha, \beta)$ if and only if $J_{s, b} f(z) \epsilon k-U M(\alpha, \beta)$.

Clearly, for $s=0$ the classes $k-U M_{b}^{s}(\alpha, \beta)$ and $k-U M(\alpha, \beta)$ coincides.

\section{Preliminaries}

Lemma 2.1. [12] Let $\hbar$ be an analytic function on $\bar{\mho}$ except for at most one pole on $\partial \mho$ and univalent on $\bar{\mho}, \wp$ be an analytic function in $\mho$ with $\wp(0)=\hbar(0)$ and $\wp(z) \neq \wp(0), z \in \mho$. If $\wp$ is not subordinate to $\hbar$, then there exist points $z_{0} \in \mho, \xi_{0} \in \partial \mho$ and $\varepsilon \geq 1$ for which

$$
\wp\left(|z|<\left|z_{0}\right|\right) \subset \hbar(\mho), \quad \wp\left(z_{0}\right)=\hbar\left(\xi_{0}\right), \quad z_{0} \wp^{\prime}\left(z_{0}\right)=\varepsilon \xi_{0} \wp^{\prime}\left(\xi_{0}\right) .
$$

Lemma 2.2. [6] If $f \in S^{*}(\alpha)$ for some $\alpha \in\left[\frac{1}{2}, 0\right)$, then

$$
\operatorname{Re}\left(\frac{f(z)}{z}\right)>\frac{1}{3-2 \alpha}
$$

Lemma 2.3. [6] If $\operatorname{Re}\left(\sqrt{f^{\prime}(z)}\right)>\alpha$ for some $\alpha \in\left[\frac{1}{2}, 0\right]$, then

$$
\operatorname{Re}\left(\frac{f(z)}{z}\right)>\frac{2 \alpha^{2}+1}{3}
$$

\section{Main Results}

Theorem 3.1. Let $k \in[0, \infty)$ and $\alpha, \beta \in[0,1]$. Also, let $p$ be a function analytic in the unit disk such that $p(0)=1$. If

$$
\operatorname{Re}\left[p(z)+\alpha \frac{z p^{\prime}(z)}{p(z)}\right]-k\left|p(z)-1+\beta \frac{z p^{\prime}(z)}{p(z)}\right|>0
$$

then

$$
p(z) \prec \frac{1+(1-2 \gamma) z}{1-z}:=h(z)
$$

where $\gamma=\gamma(k, \alpha, \beta)$ is given by

$$
\gamma(k, \alpha, \beta)=\frac{1}{4}\left[\sqrt{\frac{(\alpha-2 k+\beta k)^{2}}{(1+k)^{2}}+\frac{8(\alpha+\beta k)}{(1+k)}}-\frac{(\alpha-2 k+\beta k)}{(1+k)}\right] .
$$

Proof. We may assume that $\gamma \geq \frac{1}{2}$ since the condition $\operatorname{Re}\left(p(z)+\frac{z p^{\prime}(z)}{p(z)}\right)>0$ implies at least $\operatorname{Re}(p(z))>\frac{1}{2}$. (See [11]). Suppose now, on the contrary that $p \nprec h$. Then, by Lemma 2.1, there exist $z_{0} \in \mho, \xi_{0} \in \partial \mho$ and $m \geq 1$ such that 


$$
p\left(z_{0}\right)=\gamma+i x, z_{0} p^{\prime}\left(z_{0}\right)=m y, \text { where } y \leq-\frac{(1-\gamma)^{2}+x^{2}}{2(1-\gamma)},(x, y \in \mathbb{R}) .
$$

Using these relations, we have

$$
\operatorname{Re}\left[p\left(z_{0}\right)+\alpha \frac{z_{0} p^{\prime}\left(z_{0}\right)}{p\left(z_{0}\right)}\right]-k\left|p\left(z_{0}\right)-1+\beta \frac{z_{0} p^{\prime}\left(z_{0}\right)}{p\left(z_{0}\right)}\right|>0,
$$

or

$$
\begin{gathered}
0<\operatorname{Re}\left[p\left(z_{0}\right)+\alpha \frac{z_{0} p^{\prime}\left(z_{0}\right)}{p\left(z_{0}\right)}\right]-k\left|p\left(z_{0}\right)-1+\beta \frac{z_{0} p^{\prime}\left(z_{0}\right)}{p\left(z_{0}\right)}\right| \\
=\operatorname{Re}\left[\gamma+i x+\alpha \frac{m y}{\gamma+i x}\right]-k\left|\gamma+i x-1+\beta \frac{m y}{\gamma+i x}\right| \\
=\gamma+\frac{\alpha m y \gamma}{\gamma^{2}+x^{2}}-k\left|\frac{(\gamma+i x)^{2}-(\gamma+i x)+\beta m y}{\gamma+i x}\right| \\
\leq \gamma-\frac{\alpha \gamma}{2(1-\gamma)}\left(\frac{(1-\gamma)^{2}+x^{2}}{\gamma^{2}+x^{2}}\right)-k \frac{\sqrt{\left(X+Y x^{2}\right)^{2}+T x^{2}}}{\gamma^{2}+x^{2}}=R(x),
\end{gathered}
$$

where $X=\frac{(2 \gamma+\beta)(1-\gamma)}{2}, Y=\frac{2(1-\gamma)+\beta}{2(1-\gamma)}$ and $T=(2 \gamma-1)^{2}$. The function $R(x)$ is even in regard of $x$. Now we have to show that $R(x)$ has maximum value at $x=0$ when $\alpha, \beta \in[0,1]$ and $\gamma \in\left[\frac{1}{2}, 1\right)$. We can easily check

$$
R^{\prime}(x)=-x\left[\frac{\alpha \gamma(2 \gamma-1)}{(1-\gamma)\left(\gamma^{2}+x^{2}\right)}-k\left\{2 Y\left(X+Y x^{2}\right)+T-\frac{2 \sqrt{\left(X+Y x^{2}\right)^{2}+T x^{2}}}{\gamma^{2}+x^{2}}\right\}\right] .
$$

Then $R^{\prime}(x)=0$, if and only if, $x=0$. Since $\alpha, \beta \in[0,1]$ and $\gamma \in\left[\frac{1}{2}, 1\right)$. So one can see

$$
R^{\prime \prime}(x)=-\left[\frac{\alpha(2 \gamma-1)}{\gamma(1-\gamma)}-\frac{k}{2}\left\{(2(1-\gamma)+\beta)(2 \gamma+\beta)+2(2 \gamma-1)^{2}\right\}\right]<0 .
$$

Thus $R(x)$ has maximum value at $x=0$, that is

$$
R(x) \leq R(0)=\gamma-\frac{\alpha \gamma(1-\gamma)}{2 \gamma}-\frac{k(1-\gamma)(2 \gamma+\beta)}{2 \gamma}=0,
$$

for $\gamma=\gamma(k, \alpha, \beta)$ is given by (3.1), which contradicts the assumption. Hence

$$
p(z) \prec \frac{1+(1-2 \gamma) z}{1-z}:=h(z),
$$

where $\gamma=\gamma(k, \alpha, \beta)$ is given by (3.1).

Theorem 3.2. Let $\alpha, \beta \in[0,1], k \in[0, \infty), b \in \mathbb{C} \backslash \mathbb{Z}_{0}^{-}$and $s \in \mathbb{C}$. Then

$$
k-U M_{b}^{s}(\alpha, \beta) \subset S_{s, b}^{*}(\gamma),
$$

where $\gamma=\gamma(k, \alpha, \beta)$ is given by (3.1). 
Proof. Let $f \in k-U M_{b}^{s}(\alpha, \beta)$. Then, by Definition 1.2, $J_{s, b} f(z) \in k-U M(\alpha, \beta)$, that is

$$
\operatorname{Re}\left[(1-\alpha) \frac{z\left(J_{s, b} f(z)\right)^{\prime}}{J_{s, b} f(z)}+\alpha \frac{\left(z\left(J_{s, b} f(z)\right)^{\prime}\right)^{\prime}}{\left(J_{s, b} f(z)\right)^{\prime}}\right]>k\left|(1-\beta) \frac{z\left(J_{s, b} f(z)\right)^{\prime}}{J_{s, b} f(z)}+\beta \frac{\left(z\left(J_{s, b} f(z)\right)^{\prime}\right)^{\prime}}{\left(J_{s, b} f(z)\right)^{\prime}}-1\right|, \quad(z \in \mho) .
$$

Putting $p(z)=\frac{z\left(J_{s, b} f(z)\right)^{\prime}}{J_{s, b} f(z)}$, we have

$$
\operatorname{Re}\left[p(z)+\alpha \frac{z p^{\prime}(z)}{p(z)}\right]-k\left|p(z)-1+\beta \frac{z p^{\prime}(z)}{p(z)}\right|>0
$$

Our required result follows easily by using Theorem 3.1.

When $s=0$, then we have the following new result for class $k-U M(\alpha, \beta)$

Theorem 3.3. Let $k \in[0, \infty)$ and $\alpha, \beta \in[0,1]$. Then

$$
k-U M(\alpha, \beta) \subset S^{*}(\gamma),
$$

where $\gamma=\gamma(k, \alpha, \beta)$ is given by (3.1).

The proof is straight forward by putting $p(z)=\frac{z f^{\prime}(z)}{f(z)}$ and using Theorem 3.1.

When $k=0$, then we have the following result for a class $0-U M(\alpha, \beta)=M_{\alpha}$, introduced by Mocanu [13].

Corollary 3.1. Let $f \in M_{\alpha}$. Then $f \in S^{*}(\gamma)$, where

$$
\gamma(\alpha)=\frac{-\alpha+\sqrt{\alpha^{2}+8 \alpha}}{4}
$$

When $\beta=0$, then we have the following result, proved in [21].

Corollary 3.2. Let $f \in k-U M(\alpha, 0)=k-U M_{\alpha}$. Then $f \in S^{*}(\gamma)$, where

$$
\gamma(\alpha, k)=\frac{(2 \vartheta-\eta)+\sqrt{(2 \vartheta-\eta)^{2}+8 \eta}}{4},
$$

where $\vartheta=\frac{k}{k+1}, \eta=\frac{\alpha+k}{k+1}$.

When $\alpha=1, \beta=0$, then we have the following result, proved in [22].

Corollary 3.3. Let $f \in k-U M(1,0)=k-M N$. Then $f \in S^{*}(\gamma)$, where

$$
\gamma(k)=\frac{1}{4}\left[\sqrt{\left(\frac{1-2 k}{1+k}\right)^{2}+\frac{8}{(1+k)}}-\left(\frac{1-2 k}{1+k}\right)\right] .
$$

When $\alpha=1, \beta=0$, and $k=1$, then we have the following result, proved in [22].

Corollary 3.4. Let $f \in 1-U M(1,0)=M N$. Then $f \in S^{*}(\gamma)$, where $\gamma \simeq 0.6403$.

When $\alpha=\beta=1$, then we have the following result, proved in [6]. 
Corollary 3.5. Let $f \in k-U M(1,1)=k-U C V$. Then $f \in S^{*}(\gamma)$, where

$$
\gamma(k)=\frac{1}{4}\left[\sqrt{\left(\frac{1-k}{1+k}\right)^{2}+8}-\left(\frac{1-k}{1+k}\right)\right] .
$$

Theorem 3.4. Let $f \in k-U M_{b}^{s}(\alpha, \beta)$. Then

$$
\frac{J_{s, b} f(z)}{z} \prec \frac{1+(1-2 \eta) z}{1-z},
$$

where $\eta=\frac{1}{3-2 \gamma}$ and $\gamma=\gamma(k, \alpha, \beta)$ is given by (3.1).

Proof. Let $f \in k-U M_{b}^{s}(\alpha, \beta)$. Then by Theorem 3.2 we have

$$
\frac{z\left(J_{s, b} f(z)\right)^{\prime}}{J_{s, b} f(z)} \prec \frac{1+(1-2 \gamma) z}{1-z}
$$

where $\gamma=\gamma(k, \alpha, \beta)$ is given by (3.1). Using Lemma 2.2, we get

$$
\frac{J_{s, b} f(z)}{z} \prec \frac{1+(1-2 \eta) z}{1-z},
$$

where $\eta=\frac{1}{3-2 \gamma}$.

When $s=0$, then one can prove the following result by using Theorem 3.3 together with Lemma 2.2.

Theorem 3.5. Let $f \in k-U M(\alpha, \beta)$. Then

$$
\frac{f(z)}{z} \prec \frac{1+(1-2 \eta) z}{1-z},
$$

where $\eta=\frac{1}{3-2 \gamma}$ and $\gamma=\gamma(k, \alpha, \beta)$ is given by (3.1).

When $\alpha=1, \beta=0$, then we have the following result, proved in [22].

Corollary 3.6. Let $f \in k-U M(1,0)=k-M N$. Then

$$
\frac{f(z)}{z} \prec \frac{1+(1-2 \eta) z}{1-z},
$$

where $\eta=\frac{1}{3-2 \gamma}$ and $\gamma=\gamma(k)$ is given by (3.4).

When $\alpha=1, \beta=0$ and $k=1$, then we have the following result, proved in [22].

Corollary 3.7. Let $f \in 1-U M(1,0)=M N$. Then

$$
\frac{f(z)}{z} \prec \frac{1+(1-2 \eta) z}{1-z}, \text { where } \eta \simeq 0.58159 .
$$

When $\alpha=\beta=1$, then we have the following result, proved in [6].

Corollary 3.8. Let $f \in k-U M(1,1)=k-U C V$. Then

$$
\frac{f(z)}{z} \prec \frac{1+(1-2 \eta) z}{1-z},
$$

where $\eta=\frac{1}{3-2 \gamma}$ and $\gamma=\gamma(k)$ is given by (3.5). 
When $\alpha=\beta=k=1$, then we have the following result, proved in [6].

Corollary 3.9. Let $f \in 1-U M(1,1)=1-U C V$. Then

$$
\operatorname{Re}\left(\frac{f(z)}{z}\right)>0.6289
$$

Theorem 3.6. Let $\alpha, \beta \in[0,1], k \in[0, \infty), b \in \mathbb{C} \backslash \mathbb{Z}_{0}^{-}$and $s \in \mathbb{C}$. If

$$
\operatorname{Re}\left[\sqrt{\left(J_{s, b} f(z)\right)^{\prime}}+\alpha \frac{z\left(J_{s, b} f(z)\right)^{\prime \prime}}{2\left(J_{s, b} f(z)\right)^{\prime}}\right]>k\left|\left(J_{s, b} f(z)\right)^{\prime}+\beta \frac{z\left(J_{s, b} f(z)\right)^{\prime \prime}}{2\left(J_{s, b} f(z)\right)^{\prime}}-1\right|,
$$

then

$$
\sqrt{\left(J_{s, b} f(z)\right)^{\prime}} \prec \frac{1+(1-2 \gamma) z}{1-z} \Rightarrow \frac{J_{s, b} f(z)}{z} \prec \frac{1+(1-2 \eta) z}{1-z}
$$

where $\eta=\frac{2 \gamma^{2}+1}{3}$ and $\gamma=\gamma(k, \alpha, \beta)$ is given by (3.1).

Proof. If we put $p(z)=\sqrt{\left(J_{s, b} f(z)\right)^{\prime}}$, then

$$
\frac{z p^{\prime}(z)}{p(z)}=\frac{z\left(J_{s, b} f(z)\right)^{\prime \prime}}{2\left(J_{s, b} f(z)\right)^{\prime}}
$$

The proof follows easily by using Theorem 3.1 along with Lemma 2.3.

We can deduce the following result from Theorem 3.6 by choosing $s=0$.

Theorem 3.7. Let $k \in[0, \infty)$ and $\alpha, \beta \in[0,1]$. If

$$
\operatorname{Re}\left[\sqrt{f^{\prime}(z)}+\alpha \frac{z f^{\prime \prime}(z)}{2 f^{\prime}(z)}\right]>k\left|\sqrt{f^{\prime}(z)}+\beta \frac{z f^{\prime \prime}(z)}{2 f^{\prime}(z)}-1\right|,
$$

then

$$
\sqrt{f^{\prime}(z)} \prec \frac{1+(1-2 \gamma) z}{1-z} \Rightarrow \frac{f(z)}{z} \prec \frac{1+(1-2 \eta) z}{1-z}
$$

where $\eta=\frac{2 \gamma^{2}+1}{3}$ and $\gamma=\gamma(k, \alpha, \beta)$ is given by (3.1).

When $\alpha=1, \beta=0$, then we have the following result, proved in [22].

Corollary 3.10. If

$$
\operatorname{Re}\left[\sqrt{f^{\prime}(z)}+\frac{z f^{\prime \prime}(z)}{2 f^{\prime}(z)}\right]>k\left|\sqrt{f^{\prime}(z)}-1\right|
$$

then

$$
\operatorname{Re}\left(\sqrt{f^{\prime}(z)}\right)>\gamma \Rightarrow \operatorname{Re}\left(\frac{f(z)}{z}\right)>\eta
$$

where $\eta=\frac{2 \gamma^{2}+1}{3}$ and $\gamma=\gamma(k)$ is given by (3.4).

When $k=1$, then we have the following result, proved in [22]. 
Corollary 3.11. If

$$
\operatorname{Re}\left[\sqrt{f^{\prime}(z)}+\frac{z f^{\prime \prime}(z)}{2 f^{\prime}(z)}\right]>\left|\sqrt{f^{\prime}(z)}-1\right|
$$

then

$$
\operatorname{Re}\left(\sqrt{f^{\prime}(z)}\right)>\gamma \simeq 0.64 \Rightarrow \operatorname{Re}\left(\frac{f(z)}{z}\right)>\eta \simeq 0.60 .
$$

For $k=0$, we have the following result, refer to [22].

Corollary 3.12. If

$$
\operatorname{Re}\left[\sqrt{f^{\prime}(z)}+\frac{z f^{\prime \prime}(z)}{2 f^{\prime}(z)}\right]>0
$$

then

$$
\operatorname{Re}\left(\sqrt{f^{\prime}(z)}\right)>\gamma \simeq 0.64 \Rightarrow \operatorname{Re}\left(\frac{f(z)}{z}\right)>\eta \simeq 0.60 .
$$

Theorem 3.8. Let $\alpha, \beta \in[0,1], k \in[0, \infty), b \in \mathbb{C} \backslash \mathbb{Z}_{0}^{-}$and $s \in \mathbb{C}$. If

$$
\operatorname{Re}\left[\frac{J_{s, b} f(z)}{z}+\alpha\left(\frac{z\left(J_{s, b} f(z)\right)^{\prime}}{J_{s, b} f(z)}-1\right)\right]>k\left|\frac{J_{s, b} f(z)}{z}+\beta\left(\frac{z\left(J_{s, b} f(z)\right)^{\prime}}{J_{s, b} f(z)}-1\right)-1\right|,
$$

then

$$
\frac{J_{s, b} f(z)}{z} \prec \frac{1+(1-2 \gamma) z}{1-z},
$$

where $\gamma=\gamma(k, \alpha, \beta)$ is given by (3.1).

The proof follows easily by substituting $p(z)=\frac{J_{s, b} f(z)}{z}$ in Theorem 3.1.

For $s=0$, we can easily deduce the following result.

Theorem 3.9. Let $k \in[0, \infty)$ and $\alpha, \beta \in[0,1]$. If

$$
\operatorname{Re}\left[\frac{f(z)}{z}+\alpha\left(\frac{z f^{\prime}(z)}{f(z)}-1\right)\right]>k\left|\frac{f(z)}{z}+\beta\left(\frac{z f^{\prime}(z)}{f(z)}-1\right)-1\right|,
$$

then

$$
\frac{f(z)}{z} \prec \frac{1+(1-2 \gamma) z}{1-z},
$$

where $\gamma=\gamma(k, \alpha, \beta)$ is given by (3.1).

When $\alpha=1, \beta=0$, then we have the following result, proved in [22].

Corollary 3.13. If

$$
\operatorname{Re}\left[\frac{z f^{\prime}(z)}{f(z)}+\frac{f(z)}{z}-1\right]>k\left|\frac{f(z)}{z}-1\right| \Rightarrow \frac{f(z)}{z} \prec \frac{1+(1-2 \gamma) z}{1-z}
$$

where $\gamma=\gamma(k)$ is given by (3.4).

When $\alpha=1, \beta=0$ and $k=1$, then we have the following result, proved in [22]. 
Corollary 3.14. If

$$
\operatorname{Re}\left[\frac{z f^{\prime}(z)}{f(z)}+\frac{f(z)}{z}-1\right]>\left|\frac{f(z)}{z}-1\right| \Rightarrow \operatorname{Re}\left(\frac{f(z)}{z}\right)>\gamma \simeq 0.64 .
$$

When $\alpha=1, \beta=0$ and $k=0$, then we have following result.

\section{Corollary 3.15. If}

$$
\operatorname{Re}\left[\frac{z f^{\prime}(z)}{f(z)}+\frac{f(z)}{z}-1\right]>0 \Rightarrow \operatorname{Re}\left(\frac{f(z)}{z}\right)>\frac{1}{2}
$$

If we substitute $p(z)=\left(J_{s, b} f(z)\right)^{\prime}$ in Theorem 3.1, then we have the following result.

Theorem 3.10. Let $\alpha, \beta \in[0,1], k \in[0, \infty), b \in \mathbb{C} \backslash \mathbb{Z}_{0}^{-}$and $s \in \mathbb{C}$. If

$$
\operatorname{Re}\left[\left(J_{s, b} f(z)\right)^{\prime}+\alpha \frac{z\left(J_{s, b} f(z)\right)^{\prime \prime}}{\left(J_{s, b} f(z)\right)^{\prime}}\right]>k\left|\left(J_{s, b} f(z)\right)^{\prime}+\beta \frac{z\left(J_{s, b} f(z)\right)^{\prime \prime}}{\left(J_{s, b} f(z)\right)^{\prime}}-1\right|,
$$

then

$$
\operatorname{Re}\left(\left(J_{s, b} f(z)\right)^{\prime}\right)>\gamma
$$

where $\gamma=\gamma(k, \alpha, \beta)$ is given by (3.1).

For $s=0$, we have the following result.

Theorem 3.11. Let $k \in[0, \infty)$ and $\alpha, \beta \in[0,1]$. If

$$
R e\left[f^{\prime}(z)+\alpha \frac{z f^{\prime \prime}(z)}{f^{\prime}(z)}\right]>k\left|f^{\prime}(z)+\beta \frac{z f^{\prime \prime}(z)}{f^{\prime}(z)}-1\right|,
$$

then

$$
\operatorname{Re}\left(f^{\prime}(z)\right)>\gamma
$$

where $\gamma=\gamma(k, \alpha, \beta)$ is given by (3.1).

When $\alpha=1, \beta=0$, then we have the following result, proved in [22].

\section{Corollary 3.16. If}

$$
\operatorname{Re}\left[f^{\prime}(z)+\frac{z f^{\prime \prime}(z)}{f^{\prime}(z)}\right]>k\left|f^{\prime}(z)-1\right| \Rightarrow \operatorname{Re}\left(f^{\prime}(z)\right)>\gamma \simeq 0.64 .
$$

When $\alpha=1, \beta=k=0$, then we have the following result.

Corollary 3.17. If $\operatorname{Re}\left[f^{\prime}(z)+\frac{z f^{\prime \prime}(z)}{f^{\prime}(z)}\right]>0$, then $\operatorname{Re}\left(f^{\prime}(z)\right)>\frac{1}{2}$. 


\section{REFERENCES}

[1] J.W. Alexander, Functions which map the interior of the unit circle upon simple regions, Ann. Math. (Ser. 2). 17 (1915), 12-22.

[2] H.A. Al-Kharsani and A. Sofo, Subordination results on harmonic k-uniformly convex mappings and related classes, Comput. Math. Appl. 59 (2010), 3718-3726.

[3] S.D. Bernardi, Convex and starlike univalent functions, Trans. Amer. Mat. Soc. 135 (1969), 429-446.

[4] J. Dziok, Classes of functions associated with bounded Mocanu variation, J. Inequal. Appl. 2013 (2013), Art. ID. 349.

[5] I.B. Jung, Y.C. Kim, and H.M. Srivastava, The Hardy space of analytic functions associated with certain one-parameter families of integral operators, J. Math. Anal. Appl. 176 (1993), 138-147.

[6] S. Kanas, Subordinations for domains bounded by conic sections, Bull. Belg. Math. Soc. Simon Stevin. 15 (2008), 589-598.

[7] S. Kanas, Techniques of the differential subordination for domains bounded by conic sections, Int. J. Math. Math. Sci. 38 (2003), 2389-2400.

[8] S. Kanas and A. Wisniowska, Conic domain and starlike functions, Rev. Roumaine Math. Pures Appl. 45 (2000), $647-657$.

[9] S. Kanas and A. Wisniowska, Conic regions and k-uniform convexity, J. Comput. Math. 105 (1999), 327-336.

[10] R.J. Libera, Some classes of regular univalent functions, Proc. Amer. Math. Soc. 16 (1965), 755-758.

[11] S.S. Miller and P.T. Mocanu, Differential subordinations and applications, Marcel Dekker, Inc. New York-Basel. 2000.

[12] S.S. Miller and P.T. Mocanu, Differential subordinations and univalent functions, Michigan Math. J. 28 (1981), 157-171.

[13] P.T. Mocanu, Une propriete de convexite generlise dans la theorie de la representation conforme, Mathematica (Cluj). 11 (1969), 127-133.

[14] K.I. Noor, On generalization of uniformly convex and related functions, Comput. Math. Appl. 61 (2011), 117-125.

[15] K.I. Noor and S. Hussain, On certain analytic functions associated with Ruscheweyh derivatives and bounded Mocanu variation, J. Math. Anal. Appl. 340 (2008), 1145-1152.

[16] K.I. Noor and S.N. Malik, On coefficient inequalities of functions associated by conic domains, Comput. Math. Appl. 62 (2011), 2209-2217.

[17] K.I. Noor and S.N. Malik, On generalized bounded Mocanu variation associated with conic domain, Math. Comput. Modell. 55 (2012), 844-852.

[18] K.I. Noor and A. Muhammad, On analytic functions with generalized bounded Mocanu variation, Appl. Math. Comput. 196 (2008), 802-811.

[19] K.I. Noor and W. Ul-Haq, On some implication type results involving generalized bounded Mocanu variations, Comput. Math. Appl. 63 (2012), 1456-1461.

[20] D. Răducanu and H.M. Srivastava, A new class of analytic functions defined by means of convolution operator involving Hurwitz-Lerch Zeta function, Integral Transforms Spec. Funct. 18 (2007), 933-943.

[21] A. Rasheed, S. Hussain, M.A. Zaighum and Z. Shareef, Analytic functions related with Mocanu class, Int. J. Anal. Appl. 16 (2018), 783-792.

[22] S. Sivasubramanian, M. Govindaraj and K. Piejko, On certain class of univalent functions with conic domains involving Sokol-Nunokawa class, U.P.B. Sci. Bull. Series A. 80 (2018), 123-134.

[23] J. Sokol and M. Nunokawa, On some class of convex functions, C. R. Math. Acad. Sci. Paris. 353 (2015), 427-431.

[24] H.M. Srivastava and A.A. Attiya, An integral operator associated with the Hurwitz-Lerch Zeta function and differential subordination. Integral Transforms Spec. Funct. 18 (2007), 207-216. 
[25] H.M. Srivastava and J. Choi, Series Associated with the Zeta and Related Functions, Dordrecht, Boston, London, Kluwer Academic Publishers, 2001. 